\title{
Decay properties for evolution-parabolic coupled systems related to thermoelastic plate equations
}

\author{
Yan $\mathrm{Liu}^{1}$, Zihan $\mathrm{Cai}^{1}$, and Shuanghu Zhang ${ }^{1}$ \\ ${ }^{1}$ Guangdong University of Finance
}

January 18, 2021

\begin{abstract}
In this paper, we consider the Cauchy problem for a family of evolution-parabolic coupled systems, which are related to the classical thermoelastic plate equations containing non-local operators. By using diagonalization procedure and WKB analysis, we derive representation of solutions in the phase space. Then, sharp decay properties in a framework of $\$ \mathrm{~L}^{\wedge} \mathrm{p}-\mathrm{L}^{\wedge} \mathrm{q} \$$ are investigated via these representations. Particularly, some thresholds for the regularity-loss type decay properties are found.
\end{abstract}

\section{Hosted file}

Liu_Cai_JMAA.pdf available at https://authorea.com/users/390143/articles/504571-decayproperties-for-evolution-parabolic-coupled-systems-related-to-thermoelastic-plateequations 